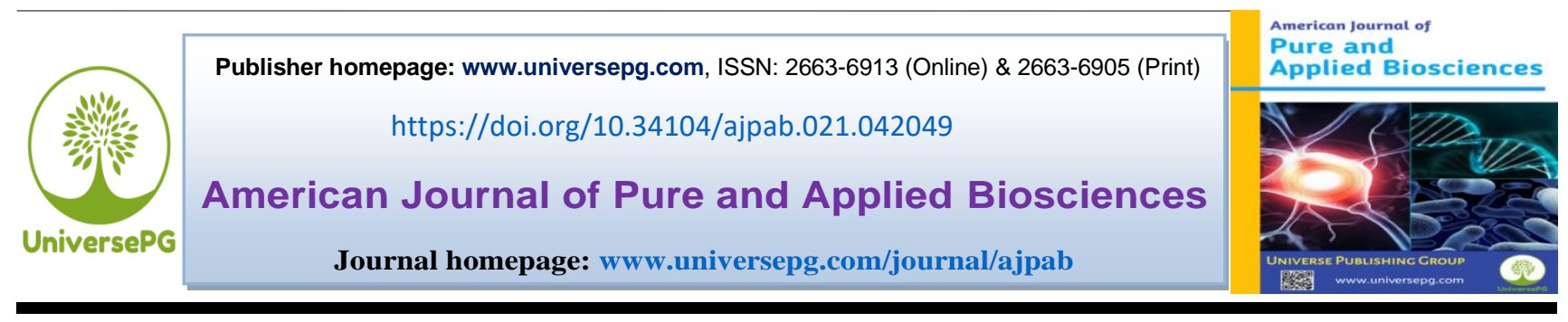

\title{
A Brief Review on the Prevalence, Diagnosis Prevention of Infectious Bovine Keratoconjunctivitis
}

\author{
Wakgari Abirham Haile ${ }^{1 *}$ and Tilahun Ayano ${ }^{2}$ \\ ${ }^{1 \& 2}$ Colleges of Veterinary Medicine and Animal Sciences, University of Gondar, Gondar, Ethiopia. \\ *Correspondence: bashirabirham@gmail.com (Wakgari Abirham Haile, Colleges of Veterinary Medicine and Animal \\ Sciences, University of Gondar, Gondar, Ethiopia).
}

\begin{abstract}
This review study is aimed to provide information about the IBK which results in ocular pain and loss of vision that would result in the reduction of the market price of the affected animal. Infectious Bovine Keratoconjunctivitis (IBK) or 'pink eye' is a common and highly contagious ocular disease. The disease is caused by the bacteria family Moraxellaceae, genus Moraxella and species Moraxella bovis. M. bovis is a gram-negative rod. The occurrence and distribution of the disease are worldwide and the persistence of the disease from year to year is by means of infected animals, which can act as carriers. Transmission is unusual in the absence of flies and occurs generally in their presence. A number of factors such as tall grass, weeds, dust, face flies and ultraviolet radiation, and other stress factors contribute to the disease occurrence. The pathogenesis of IBK is likely associated with collagenase release from epithelial cells, fibroblasts, and neutrophils. Hydrolytic enzymes of $M$. bovis possess the ability to degrade lipids, mucopolysaccharides, and matrix proteins, which may contribute to corneal ulceration. The first signs of pinkeye are characterized by excessive tearing, blinking, photophobia, and swelling of the eyelids and conjunctiva. As the disease progresses, the ocular discharge becomes purulent. The disease is usually diagnosed with the clinical signs like excessive lacrimation and culturing of the bacteria from ocular exudates. IBK is differentially diagnosed from M. bovis, Pasteurella multocida, IBRT, and Thelaziasis. Drugs may be delivered to the eye in several ways: subconjunctival injection, topical application, and systemic administration to treat the diseases. Vaccination and fly control are some of the prevention and control measures. This disease is economically very important which causes severe ocular disorder in cattle which may result in the suffering of the animal from pain and loss of vision thereby economic loss due to bodyweight loss by the stress from pain, inability to feed properly, and the blindness that reduces the price of sale. Therefore, it is recommended that the susceptible cattle's should be housed to avoid exposure to UV radiation and the populations of face flies should be controlled to minimize the incidence and transmission of IBK.
\end{abstract}

Keywords: Prevalence, Infectious Bovine keratoconjunctivitis, Moraxella bovis, Diagnosis, and Pink eye.

\section{INTRODUCTION:}

Infectious bovine keratoconjunctivitis (IBK) is a highly contagious ocular disease which is commonly known as pink eye disease. It is an economically important disease of cattle and may infect up to $80 \%$ of herd within 3 weeks. The bacterium adheres to the cells via its fimbriae and pili proteins, and produces

UniversePG I www.universepg.com $\beta$-haemolysin toxins which lyse the corneal epithelial cells (Billson et al., 2000). The disease is caused by bacterium of family moraxellaceae, genus Moraxella and species, M. bovis. M. bovis is a short, Gramnegative, non-motile rod which is usually hemolytic like E. coli; M. bovis possesses pili which assist in the adherence to epithelial cells. M. bovis can dissociate between virulent and nonvirulent forms (Kahn 
et al., 2011). IBK can also be produced by direct exposure of the eye to ultra violet (UV) light followed by the instillation of $M$. bovis. IBK presents throughout the year although there are peaks in autumn and summer (due to increased UV radiation) (Snowder et al., 2005).

The virulent forms usually possess pili and haemolysins whereas the nonvirulent do not (Punch and Slatter, 1984). Virulent M. bovis is considered to produce endotoxins, exotoxins and perhaps oculopathogenic substances (Pugh et al., 1973). The earliest signs of IBK are photophobia, blepharospasm and lacrimation, accompanied by hyperaemia of the bulbar and palpebral conjunctivae. Within one to two days a tiny corneal ulcer becomes visible and immediately afterwards, corneal edema develops and progresses as the ulcer enlarges. Neovascularization, iridocyclitis, and keratoconus may follow unless spontaneous healing or successful treatment intervenes (Rodriguez, 2006).

In addition to the etiologic agent $M$. bovis, many factors including accumulation of dust and trauma at ocular region predisposes the infection. The ability of $M$. bovis to cause the disease is influenced by host (the cattle) and environmental factors. The face fly Musca autumnalis is the important species in transmission of $M$. bovis. Moreover, the ocular and nasal discharges of infected animals can carry the pathogens, hence direct transmission from animal to animal through contact, contaminated equipment and animal handlers can also transmit the disease (Haile, 2020). The disease is characterized by increased ocular secretion, conjunctival hyperaemia, edema, corneal opacity and ulceration of the infected eyes. M. bovis possesses virulence factors that allow it to colonize the eye and result in infection. However, the current treatment and prevention measures are unrewarding and often do not circumvent the economic losses (Bedford, 1992; Ram and Steve, 2011). Based on the above backgrounds, the review of this paper was conducted with the following objectives:- To review the etiology, epidemiology and pathogenesis of IBK in cattle and to recognize the economic impacts of IBK and to identify control and prevention of IBK.

\section{Infectious Bovine Keratoconjuctivits}

Infectious bovine keratoconjunctivitis (IBK) or 'pink eye' is a common and highly contagious ocular dis- ease affecting cattle worldwide that is caused by the Gram-negative bacterium M. bovis. It produces tremensdous economic losses stem from inappetance and poor weight gain in affected animals suffering from ocular pain and visual impairment (Mcconnel et al., 2007).

\subsection{Etiology}

The disease is caused by bacteria family Moraxellaceae, genus Moraxella and species M. bovis. M. bovisa gram-negative bacillus, and is generally regarded as the etiologic agent of IBK (George and Wilson, 1984). The strains of M. bovis differ in their ability to cause clinical signs characteristic of pinkeye and are classified as virulent and virulent (Vandergaast and Rosenbusch, 1989). The virulence of the M. bovis is characterized based on morphology (rough or smooth) and by crystal violet staining. The stained colonies represent the rough morphology, which associated with virulence. It is a bacterium that is found in the eyes and nose of cattle. Depending on the strain of $M$. bovis and immunelogical response of the animal, it may not show any clinical signs upon isolation of the agent. The morphologic description of $M$. bovis colonies grown in vitro is either rough or smooth. Bacteria isolated from clinical cases of IBK form colonies that are rough, flat, dry and firm. The rough colony phenoltype is associated with cell surface pili, auto agglutination in distilled water, and hem agglutination. Following culture, rough colonies may spontaneously transform into smooth, moist colonies that do not adhere to growth media (Al-Rammahi and AlFatlawy, 2012).

The specific bacterial strain and culture conditions govern the transition from rough to smooth colonies. M. bovis isolates exhibit pleomorphism as the culture ages, occurring in pairs or chains as short, plump rods with rounded ends. On blood agar, colonies are approximately 1-3 $\mathrm{mm}$ in diameter, with a zone of beta haemolysis extending approximately $1 \mathrm{~mm}$ from the colony edge. Characteristic growth patterns occur at the interface between agar and a polystyrene petri dish, further aiding in bacterial differentiation Non hemolytic strains of $M$. bovis are not generally associated with clinical disease. Routine laboratory techniques may aid in the diagnosis of $M$. bovis is non motile and mostly hemolytic. The bacteria did not ferment carbohydrates or reduce nitrates, and is oxidase-positive (McMichael, 1992). 


\subsection{Epidemiology}

The disease occurs throughout of the world and, although it can occur in all seasons, is most common in summer and autumn. The prevalence and severity of the disease vary greatly from year to year, and it may reach epizootic proportions in feedlots and in cattle running at pasture. Only cattle are affected, the young being most susceptible, but in a susceptible population, cattle of all ages are likely to be affected. There is no mortality, and cases in which there is permanent blindness or loss of an eye are common when not treated. However, the morbidity rate can be as high as $80 \%$, with the peak infection rate at weeks 3-4 of the outbreak. Severe outbreaks can be experienced in winter, especially if the cattle are confined in close quarters such as barns or intensive feedlots (Radostitis et al., 2006).

\subsubsection{Sources of infection}

Cattle are the reservoir and the organism is carried on the conjunctiva and also in the Nares and vagina of cattle for periods exceeding 1year. Receptors for I-pili may be found on tissues other than the cornea and facilitate colonization of non-corneal tissue and unapparent infection, and the organism can switch from expression of one pilus type to the other (Radostitis et al., 2006).

\subsubsection{Transmission}

The disease is most common in summer and autumn and reaches epizootic proportions when flies and dust are abundant and grass is long; transmission is thought to be by means of these agents contaminated by the ocular and nasal discharges of infected cattle. Transmission is unusual in the absence of flies and occurs generally in their presence. The face fly $(M$. autumnalis) and Asian face fly (Musca bezzii), because of feeding preference for the area around the eyes, are important vectors. M. autumnalis is known to remain infected for periods of up to 3 days. $M$. bovis can be isolated from the crops of M. autumnalis that have fed on the eyes of infected cattle (Radostitis et al., 2006).

\subsubsection{Pre-disposing factors}

As invasion by $M$. bovis occurs, it is followed by irritation of the eye, which can be caused by a number of factors such as: tall grass, weeds, dust, face flies and ultraviolet radiation and other stress factors. The influence of tall grass, weeds and dust have been suspected, but not proven. All of the outlined factors, except UV radiation can be controlled to some extent by proper management practices. The effects of flies and UV radiation have been associated with outbreaks in several studies. Transport of cattle has been associated with the IBK carrier status of an animal, as higher numbers of the causative agents such as M. bovis, were isolated after shipment of the cattle compared with number of $M$. bovis prior to shipment (Rodriguez, 2006).

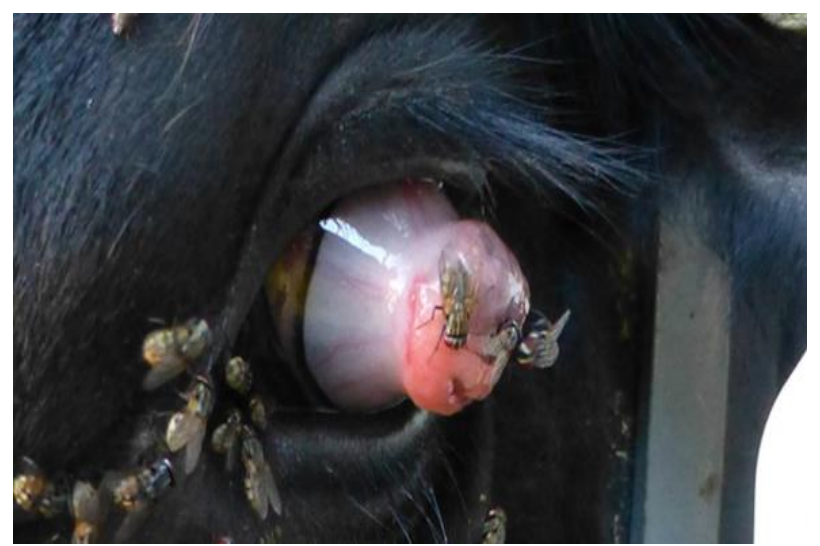

Fig 1: Flies feeding on lacrimation and transmitting the pathogen. Source: (Angelos, 2015).

\subsection{Pathogenesis}

The pathogenesis of IBK is likely associated with collagenase release from epithelial cells, fibroblasts, and neutrophils (Frank and Gerber, 1981). Hydrolytic enzymes of $M$. bovis possess the ability to degrade lipids, muco-polysaccharides, and matrix proteins, which may contribute to corneal ulceration (Brawn, 1998). The attachment of M. bovis to the corneal epithelium is mediated by the presence of pilus antigens. Q-piliated organisms are more infectious than I-piliated strains. Microscopic corneal erosions are present within 12 hours of infection and occur at this time in the absence of a significant inflammatory response, indicating that the initial production of the corneal ulceration is due to the direct cytotoxic activity of the organism. This is followed by focal loss of corneal epithelium, degeneration of keratocytes, and invasion of the corneal stroma with fibrillar destruction (Postma et al., 2008).

An inflammatory reaction occurred several days post infection and results in enlargement of the corneal ulcers with deeper stromal involvement, corneal edema, and corneal neovascularization. The lesions are localized in the eye and there is no systemic infection (Radostitis et al., 2006). 
The initial production of corneal ulcerations, however, appears to be attributed to direct cytotoxicity of $M$. bovis and not endogenous inflamematory factors from neutrophils $M$. bovis likely releases a necrotizing factor that kills corneal epithelial cells (Kagonyera and George, 1988; Gammada, 2020). The ability to produce haemolysin may be an important virulence factor of $M$. bovis. A positive correlation exists between the percentage of hemolytic strains isolated and prevalence of clinical disease (Hayle et al., 2020). Pathogenicity may be enhanced by extended time for replication and expression of virulence factors by M. bovis (Iman, 2008).

\subsection{Clinical signs}

The clinical signs of IBK are seen after the resistance of the eye is overcome by the invading organism; primarily M. Bovis (Kopeck et al., 1986; Abrar et al., 2020). The first signs of pinkeye are characterized by excessive tearing, blinking, photophobia and swelling of the eyelids, pain, keratitis, conjunctivitis, anorexia, weight loss and chronic blindness. As the disease progresses, the ocular discharge becomes purulent. Anywhere from two to five days after the initial signs are observed, cloudiness develops in the center of the cornea. This opacity extends centrifugally across the whole cornea, which leads to ulceration of the cornea and impaired vision of infected cattle (Bedford, 1976). Based on results from in-vitro assays, it's been suggested that the ulceration of the cornea is caused by direct cytotoxicity of $M$. bovis, through the release of necrotizing factors, which damage the epithelial cells of the eye (Kagonyera and George, 1988).

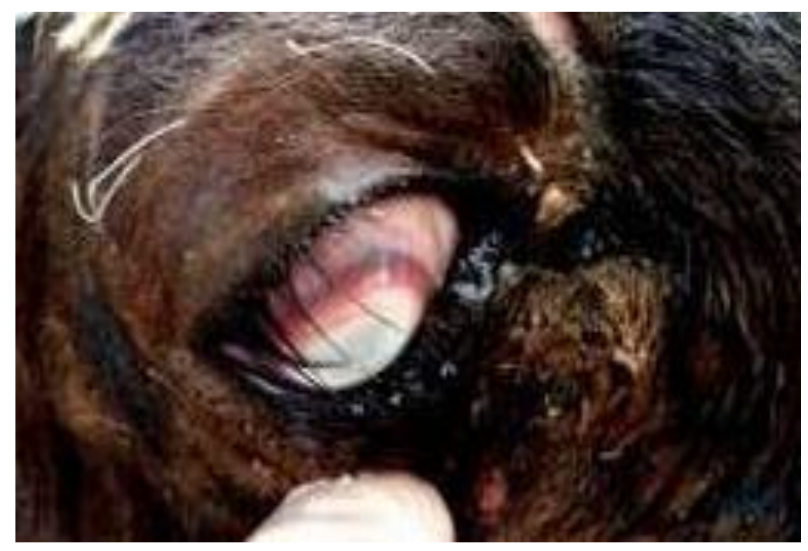

Fig 2: A calf with scleritis, keratitis and white opacity of the cornea and matting of the eye lashes with copious lacrimation. Source: (Kahn et al., 2011).

\subsection{Diagnosis}

\subsubsection{Physical diagnosis}

Pathology remains confined to the eye and does not reach the bloodstream. Diagnosis is usually based on clinical signs. On clinical examination, early disease is detectable as a raised area of cloudiness in the cornea indicating keratitis. This then spreads and corneal edema develops giving the eye a blue tint. Conjunctivitis is evident by edema, erythema and vascular congestion of the sclera vessels. Corneal ulcer may be visible on gross examination and are more obvious with administration of fluoroscin dye (https://en.wikivet.net/InfectiousBovineKeratoconju nctivitis, viewed on May 8, 2017).

\subsubsection{Microbiological cultures}

Microbiological cultures of ocular discharges or conjunctival exudates are beneficial in confirming the causative organisms. The colony of $M$. bovis has either rough or smooth morphology. The colony with rough morphology is more virulent than smooth (Angelos, 2015).

\subsubsection{Differential diagnosis}

Traumatic conjunctivitis is usually easily differentiated from IBK because of the presence of foreign matter in the eye or evidence of a physical injury. Pasteurella multocida (capsular type A) has been isolated from the eyes of housed heifers that experienced outbreaks of severe keratitis with severe loss of corneal stroma within 72 hours of onset. Mycoplasma bovis has been isolated from the eyes of steers with an outbreak of severe conjunctivitis with corneal opacity and ulceration, disease being followed by serological conversion in affected animals. Involvement of the eyelids with marked swelling was prominent. Conjunctivitis is prominent in other mycoplasmal infections that produce kerato-conjuctivitis. Infectious bovine rhinotracheitis (IB-RT), Bovine malignant catarrh and Thelaziasis are major ocular diseases which are differentially diagnosed from infectious bovine keratoconjunctivitis (Radostitis, 2006).

\subsection{Treatment}

M. bovis is susceptible to most antimicrobial agents including many antiseptics, and this combined with uncertainties concerning the etiology of the condition and a tendency for spontaneous resolution in some patients, renders the accurate assessment of 
any treatment difficult. It is generally agreed, however, that the earlier the treatment the less severe the disease and the greater the chance of controlling the outbreak (Andrews et al., 2004).

Topical: Drugs may be administered topically, by subconjunctival injection. The ideal therapy for a herd problem demands effective one-time dosage and, as such, topically applied preparations, which require frequent administration to maintain therapeutically effective levels in the Precorneal tear film (PCTF), cannot be very effective. Their short contact time is reduced further by the presence of ocular discharge and lacrimation, and blepharospasm renders their application difficult. The increased contact time as the result of specific formulation is claimed for several antibiotic preparations including cloxacillin, a penicillin and streptomycin combination (George, 1990; Rahman et al., 2019). The subconjunctival injection of antibiotics can be used as an alternative to the topical route, but the antibiotic must be placed beneath the dorsal bulbar conjuncttiva. Long-acting ampicillin, oxy tetracycline and penicillin may be used parentrally for repeated good effect. M. bovis is usually resistant to lincomycin, tylosin and erythromycin but has variable susceptibility to cloxacillin (George, 1990). Subconjunctival injections probably lead to some direct diffusion across the sclera and choroid; alternatively, the drug may gradually leak from the injection site, entering the tear film and eventually the eye via the cornea as if it were applied topically (Mcconnel, 2007).

Although M. bovis is present on the corneal and conjunctival surfaces, it colonizes the lachrymal and tarsal glands, and that any treatment must effectively penetrate these tissues. A single injection of sulphadimidine at dose rate of $100 \mathrm{mg} / \mathrm{kg}$ bodyweight will do this (Andrews et al., 2004). However, topically applied aqueous antimicrobial suspensions have a short tear halflife. Antimicrobials sprayed into the eye may prove irritating and remain only a few minutes before tears wash them away (Mcconnel, 2007).

Systemic treatment: Systemic antimicrobial therapy has been recommended as a means of targeting $M$. bovis located within lachrymal glands and nasal passages. Drugs administered systemically may enter the eye via the tear film or through the perilimbal or intraocular circulation. Generally, lipophilic drugs achieve higher intra corneal and intraocular concentrations and are more effective at penetrating the blood: tear barrier than hydrophilic drugs (Mcconnel, 2007).

Surgical treatment: Surgery can have a part to play in the treatment of IBK. The membrananictitans can be used to support the severely ulcerated cornea or protect a ruptured anterior chamber, and this technique is preferred to a temporary tarsorrhaphy in which the eyelids are sutured together. Using local anesthesia together with an auriculopalpebral nerve block to overcome any blepharospasm, the membrananictitans and the loose bulbar conjunctiva can be opposed to cover the cornea. This technique offers the advantages of directly strengthening the cornea and introducing a blood supply to the ulcer site (Andrews et al., 2004). Surgical treatment options that have been used in treating cattle with IBK include thirdeyelid flaps and tarsorrhaphy. In cases where globe rupture has occurred or where severe scarformation and globe protrusion represents a potential liability to the animal, exenteration may beindicated (Angelos, 2015)

\subsection{Prevention and control}

Infectious bovine keratoconjunctivitis can be prevented and controlled using the following techniques:-

Fly control: Fly control is an essential part of treating and preventing pinkeye. Current methods of fly control include insecticidal ear tags, sprays, pour on and charged back rubbers. Environmental insectcide programs, such as feed additives that target the larvae in the manure, are also available. The two main types of insecticidal ear tags are organophosphates and pyrethroids (http://www.thecattle site.com/articles/3922/pinkeye-in-cattle-treatme ntprevention-and-control/, viewed on May 9, 2017).

Clipping the pastures: Another aid in the prevention of IBK is to clip the pastures, if grass is too long and headed out. This will decrease much of the irritation to the cattle's eyes that can initiate the beginnings of IBK outbreak. The irritation of dust, plant pollen, or weed seeds will promote tearing from the eyes and shedding of the bacteria (M. bovis) by a few "carrier cows" in the herd. These carriers then spread the organism by contact and via face flies to the rest of the herd and susceptible animals may become infected and develop clinical IBK 
(www.vetmed.ucdavis.edu/vetext/local.../pdfs/pdfs... /cca0806-pinkeye-prevention.pdf, viewed on May 9, 2017).

Disinfectants: The routine use of a disinfectant for any equipment used on animals with pinkeye is necessary. Nolvasan (chlorhexidine) is an excellent choice because it is not irritating to tissues and works well as a disinfectant. Things to be disinfected include forceps, hemostats, or tweezers used to remove foxtails, nose tongs for restraint, or rope or nylon halters. It may be a good idea to clean and disinfect the head catch or head restraint area of the chute as it may be an area of contamination and spread of the agents (Alexander, 2010).

Vaccination: Vaccines can also be very effective in preventing pinkeye and there are a relatively large number of vaccines available which usually means no one vaccine works perfectly (www.vetmed.ucda vis.edu/vetext/local.../pdfs/pdfs.../cca0806-pinkeye prevention.pdf, viewed on May 9, 2017).

\section{The Economic Importance of IBK}

Infectious keratoconjunctivitis is a prominent disease in surveys of the predominant diseases in cattle. Loss of milk production or body condition may be caused by the discomfort, failure to feed, and temporary blindness. The conditions under which calves are reared can affect the importance of the disease. In veal calves, the disease may have no measurable effect on growth but in calves running at pasture it can result in a significant reduction of weaning weight. Occasionally, animals become completely blind and those at pasture may die of starvation (Radostitis et al., 2006).

\section{CONCLUSION AND RECOMMENDATION:}

Infectious bovine keratoconjunctivitis is a contagious bacterial disease of eye which produces great economic loss in such a way that by causing permanent blindness to cattle thereby resulting in animal trade problems. The etiological agent of the disease is the bacteria $M$. bovis Gram negative bacteria and trans-mitted from infected animal to non-infected animal mainly by face fly Musca domestica which is mechanical vector of the agent. IBK occurrence is world-wide and becomes higher in summer at which the population of flies and intensity of UV radiation increases. The early clinical signs of IBK are like excessive lacrimation, photophobia, rapid blinking and swelling of eye lids conjunctiva. IBK is a curable disease if it is recognized and treated immediately. Based on the above conclusion, the following recommendations are forwarded:-

* The susceptible cattle's should be housed to avoid exposure to UV radiation.

* The populations of face flies should be controlled to minimize the incidence and transmission of IBK.

* The animal showing the clinical signs of IBK must be diagnosed and treated soon.

- Cattle should be regularly vaccinated to immunize them against IBK.

\section{ACKNOWLEDGEMENTS:}

Firstly, we would like to thank God for his unlimited support during the whole preparation of this seminar paper. Next, we would like to acknowledge our advisor Mr. Mohammed Said for his uninterrupted advice and technical supports while preparing this seminar paper. We would like to thank the coordinators of this seminar and all my friends for their overall assistance. Finally, we would like to thank our families for their financial supports.

\section{CONFLICTS OF INTEREST:}

The author's declared there are no conflicts of interest to publish the present work.

\section{REFERENCES:}

1) Abrar A, Beyene T, and Furgasa W. (2020). Isolation, identification and antimicrobial resistance profiles of Salmonella from dairy farms in Adama and Modjo towns, central Ethiopia. Eur. J. Med. Health Sci, 2(1), 1-11. https://doi.org/10.34104/ejmhs.02001011

2) Alexander, D. (2010). Infectious bovine keratoconjunctivitis: a review of cases in clinical practice. Veterinary Clinics of North America: Food Animal Practice, 26: 487503.

https://pubmed.ncbi.nlm.nih.gov/21056797/

3) Al-Rammahi H. M. and Al-Fatlawy, H. J. (2012). Isolation and identification of conjunctival aerobic bacteria and micro flora from intact and infected eyes of cattle in Kufa district. Basrah Journal of Veterinary Research, 11(4), 1-8.

4) Andrew, A. H., Blowey, R. W., Boyd, H. and Eddy, R. G. (2004). Bovine Medicine, 
Diseases and Husbandry of Cattle. $2^{\text {nd }}$ ed. Garsington Road: Blackwell. Pp. 920-921.

5) Andrews A. H., Roger Blowey, Eddy R. G. (1992). Bovine medicine. Diseases and husbandry of cattle, Wiley-Blackwell, Oxford, 27: 712-721.

https://www.scinapse.io/papers/1570985898

6) Angelos, J. A. (2015). Infectious bovine keratoconjunctivitis (pink eye). The Veterinary Clinics of North America, 31(1): 18-46. https://doi.org/10.1016/j.cvfa.2014.11.006

7) Bedford, P. G. (1976). Infectious bovine keratoconjunctivitis. Journal of Veterinary researches, 98: 134-135.

8) Billson, F. M, Harbour, C., Michalski, W. P., Tennent, J. M., Egerton, J. R. and Hodgson, J. L. (2000). Characterization of haemolysin of Moraxella bovis using a haemolysin-neutralizing monoclonal antibody. Journal of Infection and Immuninity, 21: 3469-3474.

9) Brown, M. H., Brightman, A. H.Fenwick, B. W. and M. A. Rider, (1998). "Infectious bovine keratoconjunctivitis: a review, "Journal of Veterinary Internal Medicine, 12: 259-266.

10) Gammada I. (2020). Assessment on economic losses due to animal health and production constraints in Jimma town intensive dairy farms, Jimma, Ethiopia. Eur. J. Med. Health Sci., 2(3), 52-60. https://doi.org/10.34104/ejmhs.020.052060

11) George, C. (1990). Antibiotic trials in IBK infected cattle. Journal of Clinical Microbiology. 13: 269-271.

12) George, L. and Wilson, W. (1984). Antibiotic treatment of Moraxella bovis infection in cattle. Journal of American Veterinary Medicine Association, 185: 1206-1209. https://pubmed.ncbi.nlm.nih.gov/6392258/

13) Haile WA. (2020). Impact of climate change on animal production and expansion of animal disease: a review on Ethiopia perspective. Am. J. Pure Appl. Sci., 2(3), 6476. https://doi.org/10.34104/ajpab.020.064076

14) Hayle WA, Ahmed R, and Uddin ME. (2020). Prevalence of subclinical mastitis among small ruminants and isolation of some bacterial pathogens in Jimma Town,
Ethiopia, Eur. J. Med. Health Sci., 2(6), 107124.

https://doi.org/10.34104/ejmhs.020.01070124

15) http://www.thecattlesite.com/articles/3922/pi nkeye-in-cattle-treatment-prevention-and-co ntrol/, viewed on May 9, 2017.

16) https://en.wikivet.net/Infectious_Bovine_Ke ratoconjunctivitis, viewed on May 8, 2017.

17) Iman, B. M. (2008). A study on bacteriology of the eye infections in man and animals in Khartoum state. MSc thesis, Faculty of Veterinary Medicine, University of Khartoum, Khartoum North, Sudan.

18) Kagonyera, G. M. and George, L.W. (1988). Light and electron microscopic changes in corneas of healthy and immuno-modulated calves infected with Moraxella bovis. American Journal of Veterinary Research, 49(3): 386-395.

https://pubmed.ncbi.nlm.nih.gov/2833863/

19) Kahn C.M., Line, S., Aiello, S. E., Allen, D.G., Anderson, D. P., Jeffcott, L.B., Radostits, O. M., Reeves, P. T. and Wolf, A. M. (2011): The Merck Veterinary Manual, ${ }^{\text {th }}$ Edition, W.B. Sunders Company Ltd., London. Whitehouse Station, USA. Pp. 462465.

20) Kopecky, K. E., Pugh, G. W. and McDonald, T.J. (1986). Infectious bovine keratoconjuctivitis: Contact transmission. American Journal Veterinary Research, 47: 622624.

https://europepmc.org/article/med/3963562

21) Mcconnel, C., Shum, L. and House, JK. (2007). Infectious bovine keratoconjunctivitis antimicrobial therapy. Australian Veterinary Journal, 85: 65.

22) McMichael, J. (1992). Bacterial differentiation within Moraxella bovis colonies growing at the interface of the agar medium with the petri dish. Journal of General Microbiology, 138: 2687-2695.

https://doi.org/10.1099/00221287-138-12-2687

23) Postma, G. C., Carfagnini, J. C. and Minatel, L. (2008). Moraxella bovis pathogenicity: an update. Comparative immunology, microbiology and infectious diseases, 31: 449-458.

24) Pugh, G. W., Hughes, D. E. and Schulz, V. D. (1973). The pathophysiological effects of 
Moraxella bovis toxins on cattle, mice and guinea pigs. Can J Comp Med; 37: 70-78.

https://www.ncbi.nlm.nih.gov/pmc/articles/PM C1319727/

25) Punch, P. L. and Slatter, D.H. (1984). A review of infectious bovine keratoconjuctivitis. Veterinary journal, 54: 193-207.

26) Radostitis, O. M., Gay, C. C. and Constable, P. D. (2006). Veterinary Medicine, a Text Book of the Disease of Cattle, Horse, Sheep, Pigs and Goats. $8^{\text {th }}$ ed. Toronto. Sauder Elsevier. Pp. 995-996.

27) Rahman MA, Mahmud S, Uddin ME, and Ahmed R. (2019). Isolation, identification and antibiotic sensitivity pattern of Salmonella spp. from locally isolated egg samples, Am. J. Pure Appl. Sci., 1(1), 1-11. https://doi.org/10.34104/ajpab.019.019111

28) Ram, K. and Steve, P. (2011). Infectious Keratoconjunctivitis (Pink Eye in Cattle). Animal Health Spotlight Veterinary Medicine Extension, 27: 712-721.
29) Rodriguez, J. E. (2006). Infectious Bovine Keratoconjunctivitis in Angus Cattle, MSc thesis, Iowa State University, Pp. 114. https://doi.org/10.31274/rtd-180813-11603

30) Snowder, G. D, Van Vleck, L. D, Cundiff, L. V. and Benneth, G. L. (2005). Genetic and environmental factors associated with incidence of infectious bovine keratoconjunctivitis in preweaned beef calves. Journal of Animal Science, 83: 507-518. https://doi.org/10.2527/2005.833507x

31) Vandergaast, N. and Rosenbusch, R. F. (1989). Infectious bovine keratoconjunctivitis epizootic associated with area-wide emergence of a new Moraxella bovis pilus type. American Journal of Veterinary Research, 50: 1437-1441.

https://pubmed.ncbi.nlm.nih.gov/2572190/

32) www.vetmed.ucdavis.edu/vetext/local.../pdfs /pdfs.../cca0806-pinkeye-prevention.pdf, viewed on May 9, 2017.

Citation: Haile WA, and Ayano T. (2021). A brief review on the prevalence, diagnosis prevention of infectious bovine keratoconjunctivitis, Am. J. Pure Appl. Sci., 3(2), 42-49.

https://doi.org/10.34104/ajpab.021.042049 () 